\title{
Prevalence of coronavirus disease 2019 in rheumatic patients and evaluation of the effect of disease-modifying anti-rheumatic drugs
}

\author{
Soroush Moradi ${ }^{1} \cdot$ Maryam Masoumi $^{2} \mathbb{D} \cdot$ Somayeh Mohammadi ${ }^{2} \cdot$ Jamshid Vafaeimanesh $^{3}$. \\ Mohaddeseh Mohseni ${ }^{2} \cdot$ Hossein Mahdavi $^{4} \cdot$ Armin Aryannejad $^{1}$
}

Received: 23 June 2020 / Accepted: 10 October 2020 / Published online: 31 October 2020

(c) Società Italiana di Medicina Interna (SIMI) 2020

\begin{abstract}
One of the most controversial issues among rheumatologists is the best approach to managing a rheumatic patient (RP) with coronavirus disease 2019 (COVID-19). This study aims to evaluate the prevalence of COVID-19 in RPs compared to the general population and to relatively assess the potential role of RPs' treatment regimen against COVID-19. In a cross-sectional study, all RPs with an updated medical record between December 1, 2019, and February 29, 2020, at the rheumatology clinic of Shahid Beheshti Hospital, Qom, Iran were included (as the case group), and the prevalence of COVID-19 was compared to the paired control group - individuals without RDs, randomly selected from the Qom Health Network's database. Qom was the first city in Iran in which COVID-19 was identified and spread rapidly. Both groups were paired regarding sex, age, and underlying severe conditions. The prevalence of COVID-19 was lower in RPs than the control group $(p=0.028)$. Moreover, patients who were under treatment with disease-modifying anti-rheumatic drugs (DMARDs) and biologic agents seemed to possess a lower risk for COVID-19. Two RPs died from COVID-19, both of whom had granulomatosis and polyangiitis (GPA). The prevalence of COVID-19 in the RPs was lower than the control group, which could be associated with more adherence to the quarantine and social distancing rules by RPs and stricter routine follow-ups than the general population. Besides, taking DMARDs, such as leflunomide, might possess a protective effect against severe COVID-19, probably as a result of preventing cytokine storm.
\end{abstract}

Keywords COVID-19 · SARS-CoV-2 $\cdot$ Rheumatic diseases $\cdot$ DMARDs

$\begin{array}{ll}\text { Abbreviations } & \\ \text { COVID-19 } & \text { Coronavirus disease 2019 } \\ \text { CT } & \text { Computed tomography } \\ \text { DMARD } & \text { Disease-modifying anti-rheumatic drug } \\ \text { GPA } & \text { Granulomatosis with polyangiitis } \\ \text { HRCT } & \text { High-resolution computed tomography } \\ \text { IS } & \text { Immunosuppressive } \\ \text { RD } & \text { Rheumatic disease }\end{array}$

Maryam Masoumi

mmasoomi@muq.ac.ir

Tehran University of Medical Sciences, Tehran, Iran

2 Clinical Research and Development Center, Qom University of Medical Sciences and Health Services, Qom, Iran

3 Gastroenterology and Hepatology Diseases Research Center, Qom University of Medical Sciences, Qom, Iran

4 Shahid Beheshti University of Medical Sciences, Tehran, Iran

\begin{abstract}
RP Rheumatic patient
RT-PCR Reverse-transcription polymerase chain reaction

SARS-CoV-2 Severe acute respiratory syndrome coronavirus 2

TNF Tumor necrosis factor
\end{abstract}

\section{Introduction}

The global risk for the coronavirus disease 2019 (COVID19) was assessed by World Health Organization (WHO) as very high; it was then considered as a pandemic that affected more than two million people worldwide [1]. Severe acute respiratory syndrome coronavirus 2 (SARS-CoV-2) mainly involves the lungs and shows typical patterns in the chest high-resolution computed tomography (HRCT) images, such as ground-glass opacity and consolidation [2]. There is no specific treatment option available yet for COVID-19; 
however, some potential drugs were considered to have a beneficial effect in treating patients with COVID-19, including some antibiotics and antivirals [3] as well as angiotensinconverting enzyme (ACE) inhibitors, and hydroxychloroquine, a disease-modifying anti-rheumatic drug (DMARD) [4-6].

During the outbreak, one of the most controversial issues among rheumatologists is the best approach to manage a rheumatic patient (RP) with COVID-19. Many rheumatologists state that patients' drugs should not be discontinued or reduced because they might increase the risk of disease flare-up and lead to uncontrolled rheumatic disease (RD) [7]. Nevertheless, some may consider reducing the doses of some drugs, such as prednisolone, cautiously, or even discontinue the therapy in the case of SARS-CoV-2 infection [8]. As mentioned earlier, some drugs which are commonly used in the treatment of RD has also been considered as potential candidates for COVID-19 treatment, based on recent studies, including chloroquine, hydroxychloroquine, leflunomide, tumor necrosis factor (TNF) inhibitors, Janus kinase (JAK) inhibitors, interleukin antagonists, and even corticosteroids [6, 7]. Besides, there are still controversial views on the use of the immunosuppressive (IS) drugs, as well as their potential risks in RPs during the COVID-19 outbreak.

The current study aims to firstly evaluate the prevalence of COVID-19 in RPs and compare it to the prevalence of SARS-CoV-2 infection in a group of paired individuals without any past medical history of RDs, immunodeficiencies, or immunosuppressive therapies and second, to evaluate the potential role of DMARDs in RPs against the COVID-19.

\section{Methods}

\section{Study design}

In this cross-sectional study-between December 1, 2019, and February 29, 2020 — all RPs, including patients referred to the rheumatology clinic of the Shahid Beheshti Hospital, Qom University of Medical Sciences, Qom, Iran, outpatients who were sticking to their routine follow-up visits and in-patients were initially enrolled for further evaluations. Besides, individuals residing under the Qom Health Network coverage, without a past medical history of RDs, were randomly selected as the control group based on inclusion and exclusion criteria mentioned later in the manuscript. Then, the prevalence of COVID-19 was assessed in these two major groups. Qom was the first city in Iran in which the first confirmed cases of COVID-19 were recorded. The study protocol was implemented based on the Strengthening the Reporting of Observational Studies in Epidemiology (STROBE) statement [9] and was approved by the
Qom University of Medical Sciences and Health Services' Research and Ethics Committee.

\section{Setting and data source}

The eligibility criteria comprised all out-patients of the rheumatology clinic and in-patients in the case group, both of which had an updated hospital record from December 1, 2019, to February 29, 2020. On the contrary, residents of the city of Qom, who had been called by the Qom Health Network and had a medical record in the network's database in the same period, were randomly included as the control group-both groups aged 16-80. The so-called network was run by the Ministry of Health and Medical Education of Iran in the city of Qom long before the pandemic, which aimed at recording the general health condition of families and individuals residing in its coverage area. After the emergence of COVID-19, this network started to record the prevalence and impact of COVID-19 among its coverage population. By the time of this study, individuals who had been evaluated for the COVID-19 were randomly selected from the database. Individuals suffering from severe chronic conditions, such as heart failure, renal failure, malignancies, and cirrhosis were excluded from the study.

On the other hand, for the case group, out-patient RPs who were sticking to their routine follow-up visits and had been evaluated for the COVID-19 by the Qom Health Network, as well as for in-patients, were included. The same inclusion and exclusion criteria as the control group were applied to the case group, and both groups were paired with each other based on age, sex, and the history of severe underlying conditions mentioned earlier. Data were obtained through telephone calls and surveying the signs, symptoms, exposure, and any history of the COVID-19 based on our pre-designed questionnaire. Whenever cases were symptomatic, they were recruited for a face-to-face interview to be further investigated. Besides, records from the Qom Health Network for each individual regarding the most recent hospital admission, clinical data, imaging and laboratory results, and history of the COVID-19 and treatment were collected. The patient selection from the records was blinded utilizing the record number distribution in the study period.

\section{Variables}

The demographic variables as well as the participants' history of RD, hypertension, diabetes, hyperlipidemia, thyroid disorders, pulmonary disease, and cardiovascular disorders were included. Also, patients' symptoms in the study period, in the case of the COVID-19, including cough, hemoptysis, shortness of breath, fever, chills, headache, myalgia, malaise, diarrhea, nausea, vomiting, constipation, abdominal pain, anosmia, sore throat, and sneezing were evaluated. 
Moreover, the participants' history of admission, imaging, COVID-19, and receiving any treatments for that were recorded. The subgroups for the case group were considered based on the type of their RDs and the pharmacological/ complementary treatments which they received. The diagnostic criteria for COVID-19 stood on the national guidelines of the Ministry of Health and Medical Education of Iran, mainly by the signs and symptoms and chest computed tomography (CT) scan; in the non-conclusive results of the latter, the reverse-transcription polymerase chain reaction (RT-PCR) test was considered.

\section{Bias}

The selection bias has been minimized by blinded recruitment for the control group. To reduce potential sources of bias the data were extracted after the selection of participants. The skilled staff of Qom Health Network performed the data gathering through telephone calls, while they were completely unaware of this study.

\section{Statistical methods}

The software used for statistical analysis was IBM SPSS Statistics for Windows, version 26.0 (IBM Corporation, Armonk, NY, USA). Descriptive analyses for all variables in all participants were performed and presented. To compare categorical data, Fisher's exact test was executed, and a $t$ test was used to compare the means. All confidence intervals were set to $95 \%$. Statistical significance was considered as $p<0.05$.

\section{Results}

\section{Descriptive data}

All records of the referred patients and followed-up cases of the rheumatology clinic consisting of 249 RPs after applying inclusion and exclusion criteria were included as the case group. On the other hand, the control group included 207 individuals after applying the inclusion criteria and being paired with the case group, as mentioned earlier in the manuscript.

Of 249 RPs, 194 persons were females (77.9\%), and 55 persons were males (22.1\%); among 207 participants of the control group, 157 were females (75.8\%), and 50 were men $(24.2 \%)$. No significant difference between the sex composition between the groups was found $(p=0.655)$. The mean age for the case group was $50.2 \pm 13.1$ and $47.7 \pm 14.4$ years for the control group; the unpaired $t$ test did not show a statistically significant difference regarding age $(p=0.058)$. All demographic characteristics, comorbidities, symptoms, and any history of hospital admissions and COVID-19 for the participants are summarized in Table 1. In the case of RDs, rheumatoid arthritis was the most prevalent disorder (80.3\%) followed by systemic lupus erythematosus (7.2\%), granulomatosis with polyangiitis (GPA; formerly known as Wegener's disease) (3.6\%), anti-phospholipid syndrome $(2.8 \%)$, and ankylosing spondylitis (2.4\%).

Table 1 Demographic characteristics, comorbidities, symptoms, history of hospital admission, and COVID-19 involvement for all participants

\begin{tabular}{|c|c|c|c|c|c|}
\hline \multirow[t]{2}{*}{ Variables } & \multicolumn{2}{|c|}{$\begin{array}{l}\text { Rheumatic } \\
\text { patients }\end{array}$} & \multicolumn{2}{|c|}{$\begin{array}{l}\text { Residents of } \\
\text { Qom }\end{array}$} & \multirow[t]{2}{*}{$p$ value } \\
\hline & Number & $\%$ & Number & $\%$ & \\
\hline Participants & 249 & 100 & 207 & 100 & - \\
\hline \multicolumn{6}{|l|}{ Sex } \\
\hline Female & 194 & 77.9 & 157 & 75.8 & 0.655 \\
\hline Male & 55 & 22.1 & 50 & 24.2 & \\
\hline \multicolumn{6}{|l|}{ Comorbidity } \\
\hline Hypertension & 67 & 26.9 & 36 & 17.4 & 0.018 \\
\hline Diabetes & 43 & 17.3 & 36 & 17.4 & 1.000 \\
\hline Hyperlipidemia & 37 & 14.9 & 24 & 11.6 & 0.336 \\
\hline Hypothyroidism & 43 & 17.3 & 13 & 6.3 & $<0.001$ \\
\hline Hyperthyroidism & 6 & 2.4 & 0 & 0.0 & 0.034 \\
\hline Cardiovascular disease & 29 & 11.6 & 20 & 9.7 & 0.545 \\
\hline \multicolumn{6}{|l|}{ Symptoms } \\
\hline Dry cough & 21 & 8.4 & 23 & 11.1 & 0.344 \\
\hline Productive cough & 5 & 2.0 & 2 & 1.0 & 0.464 \\
\hline Hemoptysis & 0 & 0.0 & 0 & 0.0 & 1.000 \\
\hline Shortness of breath & 19 & 7.6 & 11 & 5.3 & 0.349 \\
\hline Fever & 21 & 8.4 & 13 & 6.3 & 0.475 \\
\hline Chills & 25 & 10.0 & 11 & 5.3 & 0.080 \\
\hline Headache & 22 & 8.8 & 15 & 7.2 & 0.607 \\
\hline Myalgia & 23 & 9.2 & 19 & 9.2 & 1.000 \\
\hline Malaise & 7 & 2.8 & 23 & 11.1 & 0.001 \\
\hline Diarrhea & 2 & 0.8 & 5 & 2.4 & 0.253 \\
\hline Nausea & 13 & 5.2 & 11 & 5.3 & 1.000 \\
\hline Vomiting & 11 & 4.4 & 1 & 0.5 & 0.008 \\
\hline Constipation & 1 & 0.4 & 7 & 3.4 & 0.026 \\
\hline Abdominal pain & 11 & 4.4 & 9 & 4.3 & 1.000 \\
\hline Anosmia & 16 & 6.4 & 19 & 9.2 & 0.293 \\
\hline Sore throat & 4 & 1.6 & 5 & 2.4 & 0.738 \\
\hline Sneezing & 0 & 0.0 & 4 & 1.9 & 0.042 \\
\hline \multicolumn{6}{|l|}{ COVID-19 } \\
\hline $\begin{array}{l}\text { COVID-19 confirmed } \\
\text { cases }\end{array}$ & 9 & 3.6 & 18 & 8.7 & 0.028 \\
\hline HRCT involvement & 7 & 2.8 & 18 & 8.7 & 0.007 \\
\hline Treatment for COVID-19 & 4 & 1.6 & 17 & 8.2 & 0.001 \\
\hline Death due to COVID-19 & 2 & 0.8 & 0 & 0.0 & 0.503 \\
\hline
\end{tabular}

COVID-19 coronavirus disease 2019, HRCT high-resolution computed tomography, $R T$ - $P C R$ reverse-transcription polymerase chain reaction 
The prevalence of hypertension in the case group (26.9\%) was significantly higher $(p=0.018)$ than the control group (17.4\%). The same was recorded in the case of hypothyroidism and hyperthyroidism, both of which were significantly more prevalent among rheumatic patients compared to the control group ( $p<0.001$ and $p=0.034$, respectively). Regarding the symptoms, malaise, constipation, and sneezing were more frequent in the control group $(p=0.001$, 0.026 , and 0.042 , respectively). Vomiting, however, occurred more in the case group $(p=0.008)$ —data on the symptoms are presented in Table 1.

\section{Main results}

The data showed that 9 out of 249 RPs (3.6\%) and 18 out of 207 residents of Qom $(8.7 \%)$ were diagnosed with COVID-19 (Table 1). The prevalence in the control group was higher than the RPs $(p=0.028)$. A chest HRCT with the patterns suggestive of COVID-19 was also significantly more common in the control group (2.8 compared to $8.7 \%$; $p=0.007)$. While eight patients $(3.9 \%)$ in the control group had a positive RT-PCR for SARS-CoV-2, no positive results were obtained in the case group $(p=0.002)$. In the case of mortality, two out of the RPs $(0.8 \%)$ died because of the COVID-19; however, no deaths were recorded in the control group $(p=0.503)$. It is noteworthy that both patients who died from COVID-19 were among the patients with GPA.

In the case of treatment regimen among RPs, 202 of 249 patients $(81.1 \%)$ were receiving prednisolone, and 132 patients $(53.0 \%)$ were using methotrexate. Eight of the patients who received prednisolone $(4.0 \%)$ and two of the patients who received methotrexate $(1.5 \%)$ were diagnosed with COVID-19. Of 108 patients (43.4\%) receiving hydroxychloroquine as an immunomodulator for their RDs, three (2.8\%) had COVID-19 and manifested lung involvement. Notably, none of the 25 patients who were receiving adalimumab or infliximab (10.0\% of total patients), and none of the 24 patients who received leflunomide were diagnosed with COVID-19.

\section{Discussion}

The prevalence of COVID-19 was assessed among the RPs compared to the randomly selected control group from the general population. The results of this study indicated a higher prevalence of COVID-19 in the control group than RPs. Based on the subgroups of RDs, $80.3 \%$ of patients in the case group had rheumatoid arthritis, among which $2.8 \%$ were diagnosed with COVID-19. Two patients in the case group died because of COVID-19, both of whom suffered from GPA. Still, no one died due to COVID-19 in the control group.
The lower prevalence of COVID-19 in the RPs than the control group could be associated with two factors. It should be taken into consideration that the nature of the RDs and their treatment - consisting of IS drugs-forced the patients to adhere more strictly to the quarantine and social distancing rules; most of the patients included in our study were suggested by their rheumatologist to take precautions more seriously. Besides, taking hydroxychloroquine and leflunomide as DMARDs in the treatment of RDs might also exert a protective effect against COVID-19, as mentioned in recent studies [10, 11]. Hydroxychloroquine possessed promising in vitro assessments of its effectiveness [12], and leflunomide was determined to inhibit the $\mathrm{T}$ cell proliferation [7]; both drugs were considered candidates for the treatment of COVID-19 based on previous studies in the first months the COVID-19 was known as a pandemic. It is worth noting that $43.4 \%$ of the RPs were receiving hydroxychloroquine daily, and $9.6 \%$ were receiving leflunomide in our study, and the lower prevalence of COVID-19 in these patients might be associated with that. No doubt that more studies are needed to evaluate this probable protective effect.

Among nine patients with GPA, a rare RD, eight were immunosuppressed extensively by prednisolone and cyclophosphamide. Although this treatment regimen is typical in GPA patients, it might make the patients more susceptible to viral infections due to extensive bone marrow suppression and immunosuppression [13]. The death of two GPA patients from the COVID-19 might be associated with this extreme immune system suppression. Thus, GPA patients should be advised to adhere to the quarantine and social distancing rules more intensively than other RPs during the COVID-19 outbreak, and stricter follow-ups should be performed.

The COVID-19 is known to be associated with a cytokine storm that involves T-helper-1. A higher concentration of TNF- $\alpha$ as well as other cytokines in critically ill patients demonstrated a positive correlation between the severity of the infection and the cytokine storm [14]. In our study, RPs were mostly under treatment with DMARDs and biologic agents, which could be associated with the lower prevalence of COVID-19 among these patients, probably due to preventing patients from experiencing cytokine storm [15].

This study indeed had some limitations; the main limitation was that the assumed prevalence for COVID-19 in the general population at the time of the study in the Qom city was little yet. To address this issue, all the rheumatology clinic patients were included as much as possible and were paired with individuals without a history of RDs. Also, the primary data were obtained through telephone calls, and all the limitations associated with this data gathering method could also apply to our study. More studies are needed to be conducted on the possible effect of taking DMARDs and IS drugs in preventing patients from experiencing severe 
infections with SARS-CoV-2 to resolve the controversial views on the use of IS drugs and DMARDs in RPs during the pandemic. The experience gained from previously emerged epidemics, such as severe acute respiratory syndrome, would also be helpful.

\section{Conclusion}

The results of our study showed that COVID-19 was less prevalent among patients with RDs than the general population in the same conditions in case of being paired regarding age, sex, and serious underlying conditions. We recorded two deaths only among RPs, which occurred in RPs suffering from GDA, a rare RD. It seems that sticking more strictly to the quarantine rules and routine follow-up visits and being under treatment with DMARDs were probable influential factors associated with the lower prevalence of the COVID19 among RPs compared to the paired general population as the control group. Still, further studies with a more extensive study population need to be performed to assess DMARDs' possible protective effect against the COVID-19 in RPs.

Author contributions All authors contributed equally to this study. The authors agreed on the protocol to be implemented and the final manuscript to be published.

Funding This study was funded by Qom University of Medical Sciences and Health Services, Qom, Iran. The university had no role in any of the study steps.

Data availability All data would be available on request.

\section{Compliance with ethical standards}

Conflict of interests The authors of this study declare that there is no conflict of interests.

Ethical approval The study protocol was approved and implemented under the supervision of Qom University of Medical Sciences' Research and Ethics Committee.

Informed consent Informed consent was obtain from each participant prior to the study process.
2. Diao K, Han P, Pang T, Li Y, Yang Z (2020) HRCT imaging features in representative imported cases of 2019 novel coronavirus pneumonia. Precis Clin Med 3(1):9-13

3. Lu R, Zhao X, Li J, Niu P, Yang B, Wu H, Wang W, Song H, Huang B, Zhu N (2020) Genomic characterisation and epidemiology of 2019 novel coronavirus: implications for virus origins and receptor binding. Lancet 395(10224):565-574

4. Liu J, Cao R, Xu M, Wang X, Zhang H, Hu H, Li Y, Hu Z, Zhong W, Wang M (2020) Hydroxychloroquine, a less toxic derivative of chloroquine, is effective in inhibiting SARS-CoV-2 infection in vitro. Cell Discov 6(1):1-4

5. Pires da Rosa G, Ferreira E (2020) Therapies used in rheumatology with relevance to coronavirus disease 2019. Clin Exp Rheumatol 38(2):370

6. Sarzi-Puttini P, Giorgi V, Sirotti S, Marotto D, Ardizzone S, Rizzardini G, Antinori S, Galli M (2020) COVID-19, cytokines and immunosuppression: what can we learn from severe acute respiratory syndrome? Clin Exp Rheumatol 38(2):337-342

7. Ferro F, Elefante E, Puxeddu I, Baldini C, Bartoloni E, Baratè C, Galimberti S, Talarico R, Mosca M, Bombardieri S (2020) COVID-19: the new challenge for rheumatologists. First update. Clin Exp Rheumatol 38(3):373-382

8. Misra DP, Agarwal V, Gasparyan AY, Zimba O (2020) Rheumatologists' perspective on coronavirus disease 19 (COVID-19) and potential therapeutic targets. Clin Rheumatol 39(7):2055-2062. https://doi.org/10.1007/s10067-020-05073-9

9. Von Elm E, Altman DG, Egger M, Pocock SJ, Gøtzsche PC, Vandenbroucke JP (2007) The Strengthening the Reporting of Observational Studies in Epidemiology (STROBE) statement: guidelines for reporting observational studies. Ann Intern Med 147(8):573-577

10. Kearney J (2020) Chloroquine as a potential treatment and prevention measure for the 2019 novel coronavirus: a review.

11. Zhou D, Dai SM, Tong Q (2020) COVID-19: a recommendation to examine the effect of hydroxychloroquine in preventing infection and progression. J Antimicrob Chemother 75(7):1667-1670

12. Kapoor KM, Kapoor A (2020) Role of chloroquine and hydroxychloroquine in the treatment of COVID-19 infection-a systematic literature review. Medrxiv

13. Khan A, Elahi F, Hashmi S, Mahida K, Ingrams D (2006) Wegener's granulomatosis: a rare, chronic and multisystem disease. Surgeon 4(1):45-52

14. Huang C, Wang Y, Li X, Ren L, Zhao J, Hu Y, Zhang L, Fan G, Xu J, Gu X (2020) Clinical features of patients infected with 2019 novel coronavirus in Wuhan, China. Lancet 395(10223):497-506

15. Bashyam AM, Feldman SR (2020) Should patients stop their biologic treatment during the COVID-19 pandemic. J Dermatol Treat $19: 1-2$

Publisher's Note Springer Nature remains neutral with regard to jurisdictional claims in published maps and institutional affiliations.

\section{References}

1. Organization WH (2020) Coronavirus disease 2019 situation reports. 